\title{
WHEEL TO AXLE FOR TRAILER SAFETY
}

Leave some end play in the wheel to axle fit when replacing trailer wheels say Safety Engineers. Some operators of heavy equipment trailers tighten the wheels adjusting nut to take out end play. This creates a safety hazard by preloading the bearings. When the trailer hauls heavy equipment, the weight of the tightly fitted bearing creates excessive frictional heat. This causes the bearing and axle surfaces to expand. With no room for expansion, more frictional heat is generated causing the lubricant to melt and run off of the bearings--the dry bearings then burn up. In this dangerous condition, the wheel could come off during operation.

The following procedure is recommended when tightening a wheel that has a single adjusting nut:

Tighten the adjusting nut, at the same time turning the wheel slowly, first in one direction--then the other. When a slight bind is felt in the wheel movement, or when torque is $50 \mathrm{ft}$. lbs., back the nut off until the nearest locking holes are aligned. This should be at least 1/6th of a turn, but not more than 1/4th. Lock the adjusting nut with the wheel in this position. The wheel should rotate freely and have an end play between .001 and .010 inch. 


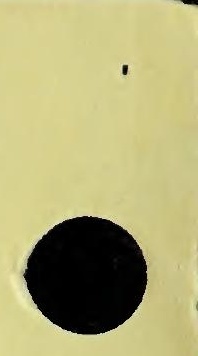

○



